\title{
Analysis on the Cryogenic Stability and Mechanical Properties of the LHD Helical Coils
}

\author{
Nagato Yanagi, Shinsaku Imagawa, Toshiyuki Mito, Andrew V. Gavrilin, Shinji Hamaguchi, Haruo Sekiguchi,
} Hirotaka Chikaraishi, Akifumi Iwamoto, Arata Nishimura, Yukio Nakamura, Takashi Satow, and Osamu Motojima

\begin{abstract}
Transient normal-transitions have been observed in the superconducting helical coils of LHD. Propagation of a normal-zone is analyzed with a numerical simulation code that deals with magnetic diffusion process in a pure aluminum stabilizer. During excitation tests, a number of spike signals are observed in the balance voltage of the helical coils, which seem to be caused by mechanical disturbances. The spike signals are analyzed by applying pulse height analysis (PHA), and the mechanical properties of the coil windings are investigated.
\end{abstract}

Index Terms-Aluminum stabilizer, dynamic stability, helical coils, LHD, numerical simulation, pulse height analysis.

\section{INTRODUCTION}

D URING the last four experimental cycles of the Large Helical Device (LHD), which is performing reactor-extrapolative plasma experiments with a helical confinement approach [1], excitation tests of the superconducting coil system have been systematically carried out. It has been observed that the pool-cooled helical coils (major radius $3.9 \mathrm{~m}$, minor radius $\sim 1$ $\mathrm{m}$ and toroidal pitch number 10) allow transient normal-transition at a current and magnetic field slightly lower than the specified operation point (current $13 \mathrm{kA}$, magnetic field $7 \mathrm{~T}$, temperature $4.4 \mathrm{~K}$ ).

For the helical windings, a composite-type superconductor is used, which consists of a NbTi/Cu Rutherford cable, a pure aluminum stabilizer, and a copper sheath with electron beam welds [2]. One of the most important issues to be noted about the specifications of this superconductor is that $\mathrm{Cu}-2 \% \mathrm{Ni}$ is used as the clad material around the pure aluminum core. This is incorporated in order to reduce the Hall current generation in an aluminum-copper composite, which significantly degrades the effective magnetoresistivity of the stabilizer and thus deteriorates cryogenic stability of the conductor [3].

The characteristics of this superconductor have been intensively studied by carrying out short sample tests as well as R\&D coil tests. It has been confirmed that the conductor becomes transiently unstable even when the transport current is lower than the steady-state "cold-end" recovery current, and an initiated normal-zone propagates over a finite length. It should be noted that the magnetic diffusion time in the pure aluminum

Manuscript received September 24, 2001.

N. Yanagi, S. Imagawa, T. Mito, S. Hamaguchi, H. Sekiguchi, H. Chikaraishi, A. Nishimura, Y. Nakamura, T. Satow, and O. Motojima are with the National Institute for Fusion Science, 322-6 Oroshi-cho, Toki, Gifu 509-5292 Japan (e-mail: yanagi@nifs.ac.jp).

A. V. Gavrilin is with the National High Magnetic Field Laboratory, Tallahassee, FL 32310 USA.

Publisher Item Identifier S 1051-8223(02)03731-4. stabilizer is relatively long due to its low resistivity. Therefore, within this characteristic time, the longitudinal resistance of the stabilizer remains considerably higher than that in the steady state, which might cause a transient degradation of cryogenic stability.

On the other hand, one also needs to pay attention to the mechanism that initiates a normal-transition. We consider that mechanical disturbances in the windings play the key role. During excitation tests, a number of spike signals are observed on the balance voltage. By analyzing these signals, it seems possible to extract useful information to understand the mechanical properties of the windings.

In this paper, discussions are given on the analysis of the cryogenic stability of the LHD helical coils in Section II, and the mechanical properties investigated by the balance voltage spike signals are described in Section III.

\section{ANALYSIS OF CRYOGENIC STABILITY}

\section{A. Observation of Normal-Transition Events}

During the excitation tests conducted up to the fourth cycle operation of LHD, normal transition events have been experienced in the helical coils nine times. Eight of these events were transient and the generated normal-zone shrank after a few seconds. However, one event, which occurred in 1998, did not recover and an emergency discharging program was activated as the quench detectors were triggered. In this event, a large amount of helium gas was vaporized due to the Joule heating as well as AC loss generation during the fast discharge (time constant of $20 \mathrm{~s}$ ). It was estimated from the signals of the temperature sensors distributed on the helical coil cans that the propagation of the generated normal-zone was finite in the longitudinal direction, and it seems probable that the final quench was triggered by deterioration of the cooling condition due to accumulation of helium bubbles at one cross-section in the coil blocks. For other normal-transition events, the generated normal-zone lasted for a few seconds ( 0.5 to $4 \mathrm{~s})$ before it naturally disappeared and the superconducting state was recovered.

The balance voltage is measured between the corresponding blocks of the two identical helical coils, H1 and H2. Each helical coil has three independent blocks, H-I (inner), H-M (middle), and $\mathrm{H}-\mathrm{O}$ (outer), and the balance voltage is obtained in such a way that the voltage signal of H1-I block is subtracted by that of H2-I block, which gives "H-I balance voltage." Fig. 1 shows an observed waveform of the H-I balance voltage when a transient normal-transition was observed in the H1-I block for about 


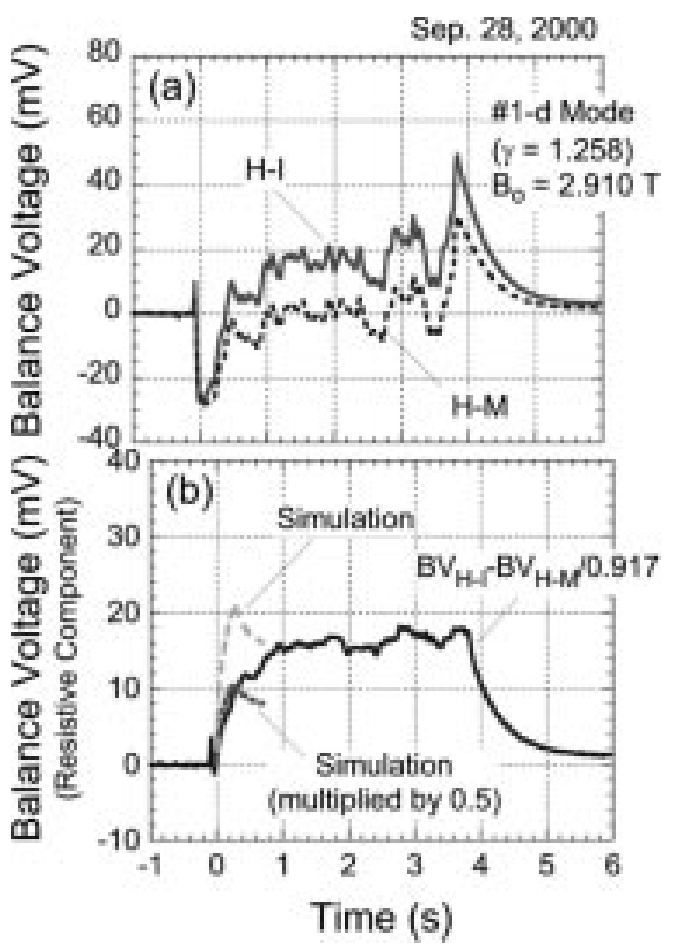

Fig. 1. Waveforms of (a) the H-I and H-M balance voltage signals observed with a normal-transition in the fourth cycle operation of LHD. The toroidal magnetic field is $2.91 \mathrm{~T}$ with the magnetic axis located at the major radius of $3.6 \mathrm{~m}$. The corresponding coil current is $10.975 \mathrm{kA}$. In (b), the resistive component of the H1-I coil voltage is extracted by subtracting the H-M balance voltage from the $\mathrm{H}-\mathrm{I}$ balance voltage. Curves obtained by numerical simulation are indicated by dashed lines.

$4 \mathrm{~s}$ in the fourth cycle operation. As is discussed in [4], a resistive component can be extracted by subtracting the $\mathrm{H}-\mathrm{M}$ balance voltage from the $\mathrm{H}-\mathrm{I}$ balance voltage with an appropriate coefficient. Here it is noted that the H-M block serves as a cancellation coil to cancel out the inductive component.

\section{B. Numerical Simulation of Normal-Zone Propagation}

A numerical simulation code has been developed to deal with normal-zone propagation in the helical coil conductor [5]. In this code, the magnetic diffusion process in the pure aluminum stabilizer is treated by incorporating the simple one-dimensional diffusion equation. Moreover, the degradation of the effective magnetoresistivity of the aluminum-copper stabilizer is included by adopting an empirical equation for Hall current generation [5].

It had been confirmed in the past that the numerical calculation well simulates the voltage waveforms which are observed in short sample tests [5] as well as in small R\&D coil tests [6]. Thus, we have tried to apply this code to analyze the normaltransition events observed in the helical coils. We have found that the present waveform cannot be easily simulated by calculation. The first problem is that the corresponding current is too low to allow a normal-transition under the same conditions for short samples. In the actual helical windings, however, the heat transfer coefficient from the conductor surface to liquid helium might be deteriorated due to the tight cooling channels between the windings. This idea is experimentally supported by heat flux measurement in which a heated surface is covered by an additional plate [7]. Thus, here we decrease the heat transfer

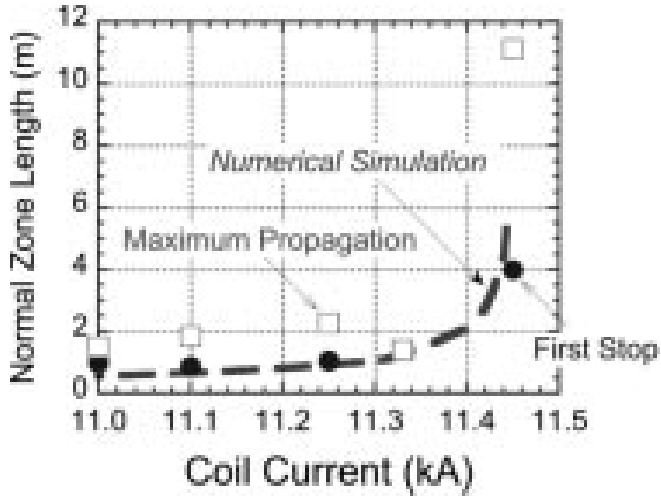

Fig. 2. Normal-zone length as a function of the coil current. The numerical simulation curve is indicated by a dashed line.

coefficient by $70 \%$ from the value that has been used for analyzing short sample tests. Then we observe a normal-transition for about 0.5 to $1 \mathrm{~s}$. The next problem is that the calculated voltage is about twice as high as the measured value. It has been observed in sample experiments that when the transport current is lower than $\sim 12 \mathrm{kA}$, an initiated normal-zone propagates only in one direction along the conductor. We cannot explain this mechanism at present, and therefore, the numerical simulation simply gives a propagation velocity twice the value that is actually observed in this current region. Thus, here we take the half value of the calculated voltage to be compared with the observed waveform. In this case, the estimated voltage seems fairly close to the measured one in the first $0.3 \mathrm{~s}$ of the propagation although the observed voltage shows a further increase after it once reaches a temporary bump, or the "first stop," at about 0.5 s. We consider that in the actual condition of the helical windings, there is a possibility that the cooling condition becomes even more deteriorated so that a generated normal-zone cannot easily recover.

From the measured voltage in a normal-transition, such as shown in Fig. 1, it is possible to estimate the normal-zone length using the typical longitudinal resistance of $1 \mu \Omega / \mathrm{m}$. The estimated normal-zone length is plotted in Fig. 2 as a function of the coil current. Here, the "first stop" corresponds to the voltage where a temporary stagnation of the balance voltage is observed at about $0.5 \mathrm{~s}$ after the initiation of a normal zone. As is seen in Fig. 2, the numerically calculated normal-zone length agrees well with the "first stop" length.

\section{Pulse Height Analysis on the Balance Voltage}

\section{A. Dependence of the Spike Energy Release on the Magnetic Field and Excitation History}

In order to investigate the mechanical properties of the helical coil windings, we take a closer look at the spike signals of the balance voltage, which are frequently observed during excitation. As is discussed in the next section, we consider that a spike signal of the balance voltage is observed when there is a mechanical displacement in a portion of the coil winding due to electromagnetic force, which enhances the coil radius and thus increase the self-inductance of the coil. In this connection, we have applied a so-called pulse height analysis (PHA) method to 

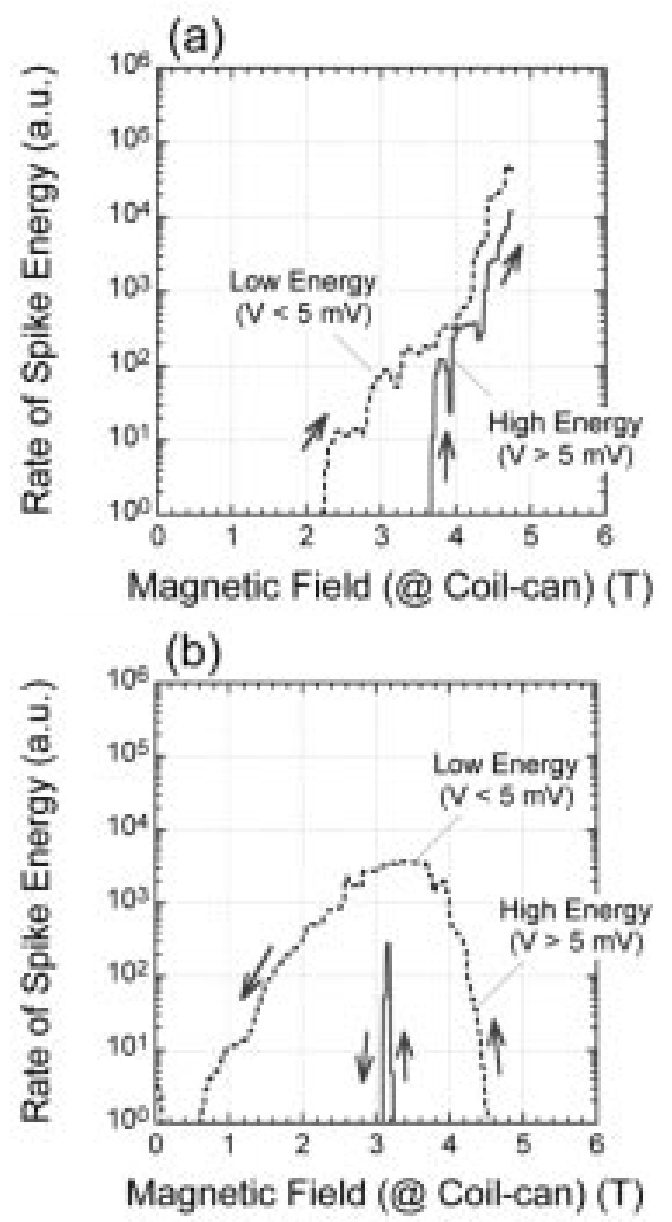

Fig. 3. Rate of the spike energy release as a function of the magnetic field (measured by a Hall probe attached to the surface of the helical coil can) during (a) ramp-up phase and (b) ramp-down phase. Low energy component and high energy component are separately shown.

the spike signals [8] in order to extract quantitative information about the mechanical disturbances in the windings. A distribution function can be obtained by counting the number of spikes in a certain range of voltage. It has been confirmed that the obtained distribution functions can be well fitted by exponential functions, which typically have two (low and high energy) components during a ramp-up phase and only one (low energy) component during a ramp-down phase [8].

If a spike voltage is multiplied by the magnetic field at the instant of the spike event, the product is a quantity that has units of power, as is further discussed in the next section. When this power is integrated in time, it gives a cumulative spike energy during excitation. This quantity is plotted as a function of the magnetic field in [8], and it was confirmed that the general trend seems very similar to that observed by acoustic emission (AE) measurement in some superconducting magnets [9], [10]. Moreover, the cumulative spike energy shows a drastic decrease from the second excitation (with a same operation condition), which is also similar to many AE measurement results in other coils.

When the obtained cumulative spike energy is differentiated by the magnetic field, it gives a rate of spike energy release per unit change of the magnetic field. An example is shown in Fig. 3

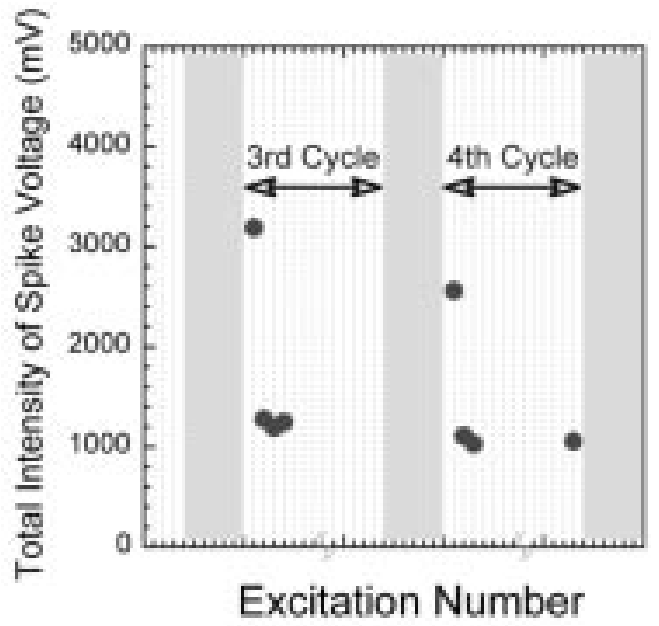

Fig. 4. Variation of the total intensity of spike voltage as a function of excitation number at the toroidal field of $2.7 \mathrm{~T}$ with the magnetic axis located at the major radius of $3.75 \mathrm{~m}$. The hatched areas indicate the warm-up and cool-down periods.

when the toroidal field of $2.7 \mathrm{~T}$ (with the magnetic axis located at the major radius of $3.75 \mathrm{~m}$ ) was first achieved in the fourth cycle operation. In Fig. 3, the rate of spike energy release is shown separately both for the low energy (pulse height lower than $5 \mathrm{mV}$ ) component and for high energy $(>5 \mathrm{mV})$ component. As is indicated by the distribution functions, the high energy component is seen dominantly in the ramp-up phase. As is also seen in Fig. 3, the rate of spike energy release with the low energy component shows a significant increase from the magnetic field (on the helical coil can) of about $2.2 \mathrm{~T}$ during the ramp-up phase, and it continuously increases as the magnetic field increases. The high energy component appears when the magnetic field reaches at about $3.6 \mathrm{~T}$, and it also continues to increase in the high field region. It should be noted that the rate of spike energy release increases up to more than four orders of magnitude during the excitation. As is discussed in the next section, we consider that thermal energy is actually released during mechanical displacements through friction between the conductor surfaces and insulating spacers. Thus, if the heat release becomes frequent, the cooling condition might be deteriorated due to possible accumulation of helium bubbles. Therefore, during real excitations, the temporal ramp-up rate is significantly lowered when we increase the current above the toroidal field of $2.6 \mathrm{~T}$. On the other hand, for the ramp-down phase, the rate of spike energy release (with the low energy component) becomes maximum at about the magnetic field of $3.5 \mathrm{~T}$. This point should be determined by the force balance between the electromagnetic force, spring force, and frictional force [10].

As is reported in [8]. the cumulative spike energy, or simply the total intensity of spike signals, drastically decreases from the second run up to the same excitation point. This indicates a clear sign of training effect. It should be noted here that the training effect seems to be partially lost by experiencing a warm-up and cool-down of the coils, as is seen in Fig. 4. On the other hand, the intensity is almost kept at constant after the second run up to the final excitation in one cycle. 


\section{B. Discussion on Winding Motion, Balance Voltage, and Conversion of Magnetic Energy}

Here we discuss the mechanism of spike signals observed in the balance voltage and the related energy release during mechanical disturbances. We consider a simplified model with two identical coils, \#1 and \#2, which are connected by a superconducting bus-line. An external power supply is connected in series to the end terminals of both coils. We consider that there is a mechanical displacement only in coil \#1, which gives a change of the self-inductance of this coil. If this change is assumed to be sufficiently smaller than the original inductance, the balance signal between the two coil voltages can be easily derived as

$$
V_{B}=I \frac{d L}{d t},
$$

where $I$ denotes the original coil current before experiencing a mechanical disturbance, and $L$ is the self-inductance of coil \#1. It is noted that if we multiply the balance voltage by the coil current, it gives a power, which is expressed as

$$
P=V_{B} \times I=I^{2} \frac{d L}{d t} .
$$

We note that it is a primary assumption that the characteristic time of a mechanical displacement is sufficiently shorter than the response time of the power supply control. This means that even if an external power supply is situated in the circuit, it is virtually equivalent to a case when the coil terminals are simply short-circuited by a superconductor. Thus, the total voltage of the two coils should always be kept zero due to the conservation of magnetic flux, and thus the coil current instantly changes when the self-inductance changes, as is expressed as

$$
\frac{d I}{d t}=-\frac{(1+2 \alpha)}{2(1+\alpha)} \frac{I}{L} \frac{d L}{d t}
$$

where $\alpha$ is a coefficient which determines the ratio between the mutual inductance and the self-inductance. Under this assumption, the rate of the magnetic energy change of the entire coils during a winding motion can be derived as

$$
\frac{d W_{m}}{d t}=-\frac{1}{2}(1+2 \alpha) I^{2} \frac{d L}{d t}=-\frac{1}{2}(1+2 \alpha) \cdot V_{B}
$$

Equation (4) means that if there is an increase in the self-inductance (due to a displacement that increases the winding radius), the total magnetic energy decreases. It might be a natural conclusion that this portion of the magnetic energy is converted to the kinetic energy of the corresponding portion of the winding while it is moving. When the winding stops to move, the energy is then converted to the elastic energy of the winding. It should also be noted that during this process, a fraction of the kinetic energy is dissipated into thermal energy due to the friction between a conductor and insulation materials. This is a very important issue to be considered from the viewpoint of cryogenic stability of the windings.

\section{SUMMARY}

Normal-transition events have been observed in the pool-cooled helical coils of LHD. A numerical analysis indicates that the generated normal-zone transiently propagates only in one direction of the conductor. Pulse height analysis has been successfully applied to extract useful information regarding the mechanical properties of the windings. The rate of magnetic energy release is increased more than four orders of magnitude during excitation.

\section{ACKNOWLEDGMENT}

The authors thank the entire staff of the LHD Device Engineering Group for their full support in conducting the excitation tests of the LHD superconducting coil system.

\section{REFERENCES}

[1] A. Iiyoshi, A. Komori, and A. Ejiri et al., "Overview of the Large Helical Device project," Nuclear Fusion, vol. 39, pp. 1245-1256, 1999.

[2] N. Yanagi, T. Mito, and S. Imagawa et al., "Development, fabrication, testing and joints of aluminum stabilized superconductors for the helical coils of LHD," in Proc. ICEC16/ICMC (Kita-Kyushu 1996), 1997, pp. $751-754$.

[3] H. Kaneko and N. Yanagi, "Enhancement of magneto-resistance due to Hall current in aluminum-copper composite," Cryogenics, vol. 32, pp. 1114-1120, 1992.

[4] S. Imagawa, N. Yanagi, and T. Mito et al., "Excitation properties and cryogenic stability of helical coils for the LHD," IEEE Trans. Appl. Supercond., vol. 11, pp. 1889-1892, 2001.

[5] A. V. Gavrilin, N. Yanagi, S. Satoh, and O. Motojima, "Computer simulation of normal zone propagation in the LHD helical coils," Advances in Supercond., vol. 11, pp. 1447-1450, 1999.

[6] N. Yanagi, S. Imagawa, and A. V. Gavrilin et al., "Analysis on the normal transition event of the LHD helical coils," IEEE Trans. Appl. Supercond., vol. 10, pp. 610-613, 2000.

[7] A. Iwamoto, T. Mito, K. Takahata, N. Yanagi, and J. Yamamoto, "Heat transfer of a large copper plate to liquid helium applicable to large scale superconductors," Cryogenics, vol. 34, pp. 321-324, 1994.

[8] N. Yanagi, S. Imagawa, and S. Hamaguchi et al., "Pulse height analysis of the spike signals on the balance voltage observed in the LHD helical coils," in Proc. ICEC18 (Mumbai 2000), 2000, pp. 179-182.

[9] O. Tsukamoto and Y. Iwasa, "Sources of acoustic emission in superconducting magnets," J. Appl. Phys., pp. 997-1007, 1983.

[10] Y. Iwasa, "Mechanical disturbances in superconducting magnets-A review,” IEEE Trans. Magn., pp. 113-120, 1992. 